\title{
Hematological and serum biochemical findings in clinical cases of cattle naturally infected with lumpy skin disease
}

\author{
Sameeh M Abutarbush ${ }^{1,2}$ \\ ${ }^{1}$ Department of Veterinary Clinical Sciences, Faculty of Veterinary Medicine, Jordan University of Science and \\ Technology, Irbid, Jordan \\ ${ }^{2}$ Veterinary Medicine Department, College of Food and Agriculture, United Arab Emirates; University, Al Ain, UAE
}

\begin{abstract}
Introduction: Lumpy skin disease (LSD) is an acute viral disease of cattle that is currently emerging in the Middle East region and poses a serious threat to Europe and the rest of the world. The objective of this study was to describe hematological and serum biochemical findings associated with natural clinical infection of LSD in cattle.

Methodology: A total of 129 animals clinically infected with LSD were enrolled in the study. Venous blood sample were collected from study animals, and hematological and serum biochemical parameters were measured.

Results: Leukocytopenia was found in $8.7 \%$, while leucocytosis was found in $18.2 \%$ of affected cattle. Decreased hematocrit concentration was seen in $18.3 \%$. Most affected cattle had reduced mean corpuscular volume (43.7\%), mean corpuscular hemoglobin (14.3\%), and mean corpuscular hemoglobin concentration (11.5\%). All cattle with abnormal platelets count had thrombocytopenia. Hyperfibrinogenemia, hyperproteinemia, and hyperalbuminemia were found in $69 \%, 59.6 \%$, and $37.2 \%$ of affected cattle, respectively. Decreased creatinine concentration was seen in $65.8 \%$. Hyperkalemia and hyperchloremia was found in $9.6 \%$ and $10.4 \%$ of the affected cattle, respectively.

Conclusions: LSD appears to be associated with inflammatory leukogram, anemia, thrombocytopenia, hyperfibrinogenemia, hyperproteinemia, decreased creatinine concentration, hyperchloramia, and hyperkalemia. These are likely due to the associated severe inflammatory process and disease complications such as anorexia and reduced muscle mass. This is the first study that documents hematological and serum biochemical findings associated with LSD infection. Understanding the blood profile picture may give further insight to the pathogenesis of the disease and help in treatment of individual cattle.
\end{abstract}

Key words: lumpy skin disease; cattle; emerging infectious diseases; hematology; biochemistry.

J Infect Dev Ctries 2015; 9(3):283-288. doi:10.3855/jidc.5038

(Received 26 March 2014 - Accepted 19 November 2014)

Copyright $\left({ }_{0} 2015\right.$ Abutarbush. This is an open-access article distributed under the Creative Commons Attribution License, which permits unrestricted use, distribution, and reproduction in any medium, provided the original work is properly cited.

\section{Introduction}

Lumpy skin disease (LSD) is an acute viral disease of cattle. It originated in Africa, and it is endemic in most African countries. LSD is currently emerging in the Middle East region and poses a serious threat to Europe and the rest of the world [1,2]. The disease is caused by lumpy skin disease virus, which belongs to the genus Capripoxvirus within the subfamily Chordopoxvirinae of the family Poxviridae [2,3].

LSD is associated with high morbidity and low mortality in cattle $[1,2]$. It causes serious economic losses due to severe reduction in milk production and feed intake as well as weight conversion. Furthermore, it causes abortion, infertility, and damaged cattle hides. LSD is considered a notifiable disease, and in affected countries, it results in serious restrictions to international trade $[1,2]$.

In natural outbreaks of the disease, the incubation period is reported to be between one and four weeks
[4,5]. Some animals show clinical signs of the disease while others do not. The first clinical signs of infection are high fever and lachrymation, and palpable lymph nodes become enlarged. Soon after the animal becomes febrile, few to several cutaneous nodules, which vary in size, appear on different regions of the body. These may cover the entire animal and, in some cases, lesions can be seen in the mouth and nose as well as the mucous membranes of the eye [2,4-7].

Post-mortem examination of the affected cattle may reveal widespread pox lesions that can be seen in each organ [7]. LSD lesions may be seen throughout the gastrointestinal and respiratory tracts. In addition to the classical skin lesions, limb edema can be seen in one or more limbs.

Characteristic signs, histopathology, and virus isolation as well as polymerase chain reaction (PCR) can all be used to diagnose LSD in affected cattle $[1,2]$. Treatment is only symptomatic and targets 
secondary bacterial infections; the best method of control is vaccination [8].

Pathogenesis of LSD in cattle has been studied before, and there is a good understanding of the way the virus causes lesions in affected cattle. In addition, clinical signs and post-mortem lesions are well described. However, no knowledge about hematological and serum biochemical findings associated with natural clinical infection of LSD is present in the veterinary literature.

The aim of this study was to describe the hematological and serum biochemical findings associated with natural clinical infection of lumpy skin disease in cattle.

\section{Methodology}

Study overview

During a lumpy skin disease natural outbreak in northern Jordan, where the disease was confirmed using PCR [9], venous blood samples were collected from clinically affected cattle. Different hematological and serum biochemical parameters were measured based on test availability.

\section{Case enrolment criteria}

All clinically affected cases were eligible for enrolment in the study. A sick animal was considered a clinical case of LSD if it was located in the disease outbreak area, had skin nodules characteristic of LSD infection (sitfasts lesions), and enlarged palpable lymph nodes. Additional clinical signs that were considered supportive included fever, pox lesions in the oral and nasal cavities, and ventral and limb edema.

\section{Blood sample collection}

Venous blood samples were collected from the jugular vein of each enrolled animal. The blood was collected in two tubes: one plain tube and one tube containing ethylenediaminetetraacetic acid (EDTA).

\section{Hematology}

Blood from the EDTA tubes was used to measure hematological parameters. Those included white blood cell count (WBC), red blood cell count (RBC), platelets, hematocrit (HCT), hemoglobin (HB), mean corpuscular volume (MCV), mean corpuscular hemoglobin $(\mathrm{MCH})$, and mean corpuscular hemoglobin concentration (MCHC). Hematological parameters were evaluated using an automatic counter (ABCTM, animal blood counter, HORIBA Medical, Montpellier, France).

\section{Serum biochemical analysis}

Blood from plain tubes was centrifuged, and the serum was harvested. Harvested serum was used to measure total protein, albumin, fibrinogen, alkaline phosphatase (ALP), total bilirubin, blood urea nitrogen (BUN), creatinine, and serum electrolyte (potassium $[\mathrm{K}]$, sodium $[\mathrm{Na}]$, and chloride $[\mathrm{Cl}]$ ) concentrations. Biochemical analyses were done spectrophotometrically using commercially available diagnostic kits (SYRBIO diagnostic reagents for laboratories, Damascus, Syria).

\section{Data management and analysis}

Hematological and serum biochemical parameters measurements were entered into a Microsoft Excel 2007 spreadsheet. SPSS software version 17 was used to do descriptive statistical analyses. In addition, the same software was used to compare the mean values of cattle with high and low measurements using the $t$ test. A p value less or equal to 0.05 was considered significant.

\section{Results}

A total of 129 cattle met the selection criteria and were enrolled in the study. They were all Holstein Frisian cattle and comprised 7 calves, 8 bulls, 7 pregnant heifers, 14 dry cows, and 93 milking cows. The ages of the enrolled cattle ranged from 5 months to 10 years $(n=124$, mean $=4.3$ years, $S D \pm 1.9$ years). The number of cattle that had abnormal hematological and serum biochemical values varied according the measured parameter can be seen in Table 1.

When considering only the number of cattle that showed abnormal hematological findings, abnormal white blood cell count was recorded in 34/126 (26.9\%) of cattle. Of those, $11(32.3 \%)$ had low, while 23 $(67.6 \%)$ had high abnormal values. Abnormal red blood cell count was recorded in 19/126 (15\%) of the cattle; of those, $9(47.4 \%)$ had low, while 10 (52.6\%) had high abnormal values. Abnormal hemoglobin concentration count was recorded in 11/126 (8.7\%) of the cattle; of those, $8(72.8 \%)$ had low, while 3 (27.2\%) had high abnormal values. Abnormal hematocrit concentration was recorded in 29/126 $(23 \%)$ of the cattle; of those, $23(79.4 \%)$ had low, while $6(20.6 \%)$ had high abnormal values. Abnormal mean corpuscular volume was recorded in 57/126 $(45.2 \%)$ of the cattle; of those, 55 (96.5\%) had low, while $2(3.5 \%)$ had high abnormal values. Abnormal mean corpuscular hemoglobin was recorded in 19/126 (15\%) of the cattle; of those, 18 (94.8\%) had low, 
while 1 (5.2\%) had high abnormal values. Abnormal mean corpuscular hemoglobin concentration was recorded in 15/122 (12.2\%) of the cattle; of those, 14 (93.4\%) had low, while 1 (6.6\%) had high abnormal values. Abnormal platelet count was recorded in $32 / 126(25.3 \%)$ of the cattle; all of them had low abnormal values.

When considering the total number of enrolled cattle, leukocytopenia was found in $8.7 \%$ while, leucocytosis was in $18.2 \%$ of the affected cattle (Table 1). A decreased red blood cell count was seen in $7.1 \%$, while an increased number was seen in $7.9 \%$. Higher number of cattle had reduced blood hemoglobin concentration $(6.4 \%)$ compared to those that had higher concentrations $(2.3 \%)$. The number of cattle that had reduced hematocrit concentration (18.3\%) was almost four times the number of cattle with increased concentration (4.7\%). Most affected cattle had reduced mean corpuscular volume $(43.7 \%)$, mean corpuscular hemoglobin (14.3\%), and mean corpuscular hemoglobin concentrations $(11.5 \%)$. All cattle with abnormal platelet counts had thrombocytopenia.

In general, hematology revealed leukocytosis or leukopenia, decreased hematocrit concentration, decreased or increased red blood cell count, decreased hemoglobin concentration, reduced mean corpuscular volume, reduced mean corpuscular hemoglobin, reduced mean corpuscular hemoglobin concentration, and thrombocytopenia.

When only considering the number of cattle that showed abnormal serum biochemical findings, abnormal total protein concentration was recorded in $91 / 129(70.4 \%)$ of the cattle (Table 2). Of those, 14 (15.4\%) had low, while 77 (84.6\%) had high abnormal values. Abnormal albumin concentration was recorded in $75 / 129(58.1 \%)$ of the cattle; of those, $27(36 \%)$ had low, while 48 (64\%) had high abnormal values.

Table 1. Haematological findings in cattle clinically affected with lumpy skin disease.

\begin{tabular}{|c|c|c|c|c|c|c|}
\hline Analate & $\begin{array}{l}\text { Reference } \\
\text { (Radostitis } \\
\text { et al, 2000) }\end{array}$ & Mean & Range & $\begin{array}{c}\text { No. of Cattle with } \\
\text { Low Abnormal } \\
\text { Values }\end{array}$ & $\begin{array}{c}\text { No. of Cattle with } \\
\text { High Abnormal } \\
\text { Values }\end{array}$ & $\begin{array}{c}\text { Comparison Between } \\
\text { the means value of } \\
\text { Cattle with High \& } \\
\text { Low Abnormal } \\
\text { Values } \\
\text { (P value) }\end{array}$ \\
\hline WBC $(\times 109 / \mathrm{L})$ & $4-12$ & 9.0 & $1.3-29.9$ & $11 / 126$ & $23 / 126$ & 0.00 \\
\hline $\mathrm{RBC}(\times 1012 / \mathrm{L})$ & $5-10$ & 7.4 & $3.2-17.3$ & $9 / 126$ & $10 / 126$ & 0.00 \\
\hline $\operatorname{MCV}(f l)$ & $40-60$ & 40.7 & $27.6-81.4$ & $55 / 126$ & $2 / 126$ & 0.00 \\
\hline $\mathrm{MCH}(\mathrm{pg})$ & $11-17$ & 12.8 & $8.3-19.4$ & $18 / 126$ & $1 / 126$ & 0.00 \\
\hline $\mathrm{MCHC}(\mathrm{g} / \mathrm{L})$ & $300-360$ & 315 & $146-367$ & $14 / 122$ & $1 / 122$ & 0.054 \\
\hline Platelets $(\times 109 / \mathrm{L})$ & $100-800$ & 207 & $35-589$ & $32 / 126$ & $0 / 126$ & N/A \\
\hline
\end{tabular}

WBC: white blood cell count; RBC: red blood cell count; HB: hemoglobin; HCT: hematocrit; MCV: mean corpuscular volume; MCH: mean corpuscular hemoglobin; MCHC: mean corpuscular hemoglobin concentration

Table 2. Serum biochemical findings in cattle clinically affected with lumpy skin disease.

\begin{tabular}{|c|c|c|c|c|c|c|}
\hline Analate & $\begin{array}{l}\text { Reference } \\
\text { (Radostitis } \\
\text { et al, 2000) }\end{array}$ & Mean & Range & $\begin{array}{c}\text { No. of Cattle with } \\
\text { Low Abnormal } \\
\text { Values }\end{array}$ & $\begin{array}{c}\text { No. of Cattle with } \\
\text { High Abnormal } \\
\text { Values }\end{array}$ & $\begin{array}{c}\text { Comparison Between } \\
\text { the means value of } \\
\text { Cattle with High \& } \\
\text { Low Abnormal } \\
\text { Values (P value) }\end{array}$ \\
\hline Total protein $(\mathrm{g} / \mathrm{L})$ & $67-75$ & 76.6 & $54-105$ & $14 / 129$ & $77 / 129$ & 0.000 \\
\hline Albumin(g/L) & $30-36$ & 35 & $20-67$ & $27 / 129$ & $48 / 129$ & 0.000 \\
\hline Fibrinogen $(\mathrm{g} / \mathrm{L})$ & $200-500$ & 625 & $400-1000$ & $0 / 126$ & $87 / 126$ & N/A \\
\hline ALP (units/L) & $0-500$ & 51 & $0-321$ & $0 / 128$ & $0 / 128$ & N/A \\
\hline $\begin{array}{l}\text { Total Bilirubin } \\
(\mu \mathrm{mol} / \mathrm{L})\end{array}$ & $0.17-8.55$ & 4.90 & $0.17-59.90$ & $0 / 118$ & $13 / 118$ & N/A \\
\hline $\begin{array}{l}\text { Blood urea nitrogen } \\
(\mathrm{mmol} / \mathrm{L})\end{array}$ & $2-7.5$ & 4.7 & $2.1-20.2$ & $0 / 129$ & $2 / 129$ & N/A \\
\hline Creatinine $(\mu \mathrm{mol} / \mathrm{L})$ & $67-175$ & 65.2 & $8.8-176.8$ & $79 / 120$ & $0 / 120$ & N/A \\
\hline $\mathrm{Na}(\mathrm{mmol} / \mathrm{l})$ & $132-152$ & 138 & $106-160$ & $16 / 124$ & $3 / 124$ & 0.000 \\
\hline $\mathrm{K}(\mathrm{mmol} / \mathrm{l})$ & $3.9-5.8$ & 4.9 & $3.6-7.5$ & $9 / 124$ & $12 / 124$ & 0.000 \\
\hline $\mathrm{Cl}(\mathrm{mmol} / \mathrm{l})$ & $95-110$ & 104 & $82-142$ & $3 / 124$ & $13 / 124$ & 0.000 \\
\hline
\end{tabular}

ALP: alkaline phosphatase; Na: sodium; K: potassium; Cl: chloride 
Abnormal fibrinogen concentration was recorded in $87 / 126(69 \%)$ of the cattle; all of them had high abnormal values. No cattle had abnormal alkaline phosphatase concentration. Abnormal total bilirubin concentration was recorded in $13 / 118(11 \%)$ of the cattle; all of them had high abnormal values. Abnormal blood urea nitrogen concentration was recorded in 2/129 (1.5\%) of the cattle; all of them had high abnormal values. Abnormal creatinine concentration was recorded in 79/120 (65.8\%) of the cattle; all of them had low abnormal values. Abnormal sodium concentration was recorded in 19/124 (15.3\%) of cattle; if those, $16(84.3 \%)$ had low, while 3 $(15.7 \%)$ had high abnormal values. Abnormal potassium concentration was recorded in 21/124 $(16.9 \%)$ of the cattle; of those, $9(42.9 \%)$ had low, while $12(57.1 \%)$ had high abnormal values. Abnormal chloride concentration was recorded in $16 / 124(12.8 \%)$ of the cattle; of those, $3(18.8 \%)$ had low, while $13(81.2 \%)$ had high abnormal values.

When considering the total number of enrolled cattle, hyperproteinemia was found in $59.6 \%$, while hypoproteinemia was in $10.8 \%$ of the affected cattle (Table 2). Hyperalbuminemia was found in $37.2 \%$, while hypoalbuminemia was in $20.9 \%$ of the affected cattle. Hyperfibrinogenemia was found in $69 \%$ of the affected cattle. Hyperbilirubinemia was seen in $11 \%$ of the affected cattle. Increased BUN concentration was seen in $1.5 \%$ of the affected cattle. Decreased creatinine concentration was seen in $65.8 \%$ of the affected cattle. Hypernatremia was found in $2.4 \%$, while hyponatremia was found in $17.9 \%$ of the affected cattle. Hyperkalemia was found in $9.6 \%$, while hypokalemia was found in $7.2 \%$ of the affected cattle. Hyperchloremia was found in $10.4 \%$, while hypochloremia was found in $2.4 \%$ of the affected cattle.

In general, serum biochemistry revealed hyper- or hypoproteinemia, hyper- or hypoalbuminemia, hyperfibrinogenemia, hyperbilirubinemia, increased BUN concentration, decreased creatinine concentration, hyponatremia, hypo- or hyperkalemia, and hyperchloremia.

Statistical comparison results between the means value of cattle with high and low measurements can be seen in Tables 1 and 2.

\section{Discussion}

This is the first study that documents hematological and serum biochemical findings associated with natural clinical infection of lumpy skin disease in cattle. The findings of this study will add to the understanding of the pathogenesis of LSD in cattle and may give more insight into the treatment of clinically affected cases.

Some hematological findings of cattle affected clinically with LSD were variable, while most were consistent. Some cattle showed leukocytosis, while others showed leukopenia. This is likely related to the stage and severity of infection. However, there are no known criteria to determine the stage of infection. In cattle, leukopenia is usually seen in the developmental stage of the acute infection, after which the production of neutrophils is intensified, leading to leukocytosis [10]. The number of cattle with leukocytosis was twice that of cattle with leukopenia, which is in agreement with the chronic and debilitating nature of LSD in cattle.

More affected cattle had increased red blood cell counts. This can be absolute, due to erythropoiesis, or relative due to dehydration. Both causes are equally possible, since affected cases usually suffer from dehydration enhanced by fever, anorexia, and lethargy, which are commonly seen in LSD infection. In addition, chronic diseases in large animals can be associated with absolute erythrocytosis [11].

In this study, most affected cattle had anemia characterized by decreased hematocrit concentration, $\mathrm{MCV}, \mathrm{MCH}$, and MCHC. These findings suggest that affected cattle suffer from anemia of chronic disease, which is very likely in LSD infection [12]. The chronic inflammatory response in this type of anemia causes disturbance of iron metabolism. Iron deficiency results in decreased $\mathrm{MCV}, \mathrm{MCH}$, and $\mathrm{MCHC}$. This type of anemia is usually mild and slowly progressive [12]. In addition, it is usually associated with leukocytosis, hyperfibrinogenemia, and hyperproteinemia, as seen in some of the affected cattle in this study.

Thrombocytopenia was seen in all of the affected cattle with abnormal platelet counts. Decreased platelet count can be caused by decreased platelet production, due to bone marrow abnormality or platelet sequestration, which occurs in splenomegaly and non-infectious inflammatory disorders [13]. In cattle, the most common cause of thrombocytopenia is shortened platelet life span. This is usually caused by excessive platelet consumption due to disseminated intravascular coagulation (DIC), overwhelming endotoxemia, and systemic vasculitides. The latter is the likely cause of thrombocytopenia in this study since widespread vasculitis, due to LSD virus tropism for endothelial cells, is a characteristic histopathological finding in affected cases [14]. 
Serum biochemical findings were variable in the affected cattle. Most of the cattle with abnormal total protein measurement had hyperproteinemia. Hyperproteinemia in the current study can be part of the inflammatory response and/or caused by dehydration. Severe inflammatory process as a cause of hyperproteinemia is supported by the inflammatory leukogram present and significant hyperfibrinogenemia. The latter is nearly always associated with severe inflammatory process in cattle. Measuring plasma globulins would have helped in supporting or refuting this possibility; unfortunately, it was not done in this study. Dehydration is also a possible cause of hyperproteinemia in light of the presence of hyperalbuminemia in some of the affected cattle. Hyperproteinemia caused by dehydration is usually associated with increased hematocrit values. However, as in most of the affected cattle in this study, dehydration in anemic animals is associated with normal or subnormal HCT values [15]. Therefore, if dehydration is the main cause of hyperproteinemia, the number of cattle suffering from anemia is underestimated, and more affected cattle with normal HCT values are, in fact, anemic.

Hyperbilirubinemia was seen in some affected cases and it was likely secondary to the systemic disease process. Decreased creatinine concentration was seen in all affected cattle with abnormal creatinine concentration measurement. Anorexia and loss of muscle mass, which are known to occur in LSD infection, result in reduced serum creatinine levels [16].

Hyponatremia was seen in some of the affected cattle. It is usually associated with a condition that leads to sodium depletion, such as vomiting or diarrhea. Those are not known features of LSD infection in cattle. However, in light of the hyperproteinemia that is present in affected cases, it is likely that hyponatremia is a false finding [16]. Anorexia was the likely cause of hypokalemia in some of the affected cattle, while hyperkalemia may have been caused by the muscular damage that is associated with LSD infection $[14,16]$. Some of the affected cattle had hyperchloremia, which is likely proportional and caused by decreased water intake [16].

Lumpy skin disease is associated with variable changes in hematology and serum biochemistry. The changes are mainly caused by the primary disease process, and the differences seen within the same parameter are likely due to the stage of the diseases and the complexity of the case.

\section{Conclusions}

Inflammatory

thrombocytopenia,

leukogram, anemia, hyperfibrinogenemia, hyperproteinemia, decreased creatinine concentration, hyperkalemia, and hyperchloremia are the main findings in clinical cases of LSD in cattle. These are likely due to the associated severe inflammatory process and disease complications, such as anorexia and reduced muscle mass. Understanding the blood profile picture of cattle affected by LSD gives further insight into the pathogenesis of the disease and can help in the treatment of individual cattle.

\section{References}

1. Davies FG (1991) Lumpy skin disease of cattle: a growing problem in Africa and the Near East. World Animal Review 68: $37-42$.

2. Tuppurainen ES, Oura CA (2012) Review: lumpy skin disease: an emerging threat to Europe the Middle East and Asia. Transbound Emerg Dis 5940-5948.

3. Buller RM, Arif BM, Black DN, Dumbell KR, Esposito JJ, Lefkowitz EJ, McFadden G, Moss B, Mercer AA, Moyer RW, Skinner MA, Tripathy DN (2005) Poxviridae. In Fauquet CM, Mayo MA, Maniloff J, Desselberger U, Ball LA, editors. Virus Taxonomy: Eight Report of the International Committee on the Taxonomy of Viruses. Oxford: Elsevier Academic Press. 117-133.

4. Haig DA (1957) Lumpy skin disease. Bulletin of Epizootic Diseases of Africa 5: 421-430

5. Coetzer JAW (2004) Lumpy skin disease In: Coetzer JAW and Tustin RC, editors. Infectious Diseases of Livestock, 2nd edition. Oxford: University Press Southern Africa. 12681276.

6. Thomas AD, Mare CvE (1945) Knopvelsiekte. J S Afr Vet Assoc 16: 36-43

7. Babiuk S, Bowden TR, Parkyn G, Dalman B, Manning L, Neufeld J, Embury, Hyatt C, Copps J, Boyle DB (2008) Quantification of lumpy skin disease virus following experimental infection in cattle. Transbound Emerg Dis 55: 299-307.

8. Hunter P, Wallace D (2001) Lumpy skin disease in southern Africa: a review of the disease and aspects of control. J S Afr Vet Assoc 72: 68-71.

9. International Society for Infectious Diseases, ProMED-Mail (2013) Lumpy skin disease bovine - Jordan. (IRAM) OIE 201306121768278. Available: http://www.promedmail.org. Accessed 5 August 2013.

10. Morris DD (2002) Alterations in the leukogram. In Smith BP, editor. Large Animal Internal Medicine, 2nd edition. New York: Mosby. 420-426.

11. Morris DD (2002) Alterations in the Erythron. In Smith BP, editor. Large Animal Internal Medicine, 2nd edition. New York: Mosby. 415-419.

12. Carlson GP (2002) Depression anemia. In Smith BP, editor. Large Animal Internal Medicine, 2nd edition. New York: Mosby. 1063-1065.

13. Morris DD (2002) Alterations in the clotting profile. In Smith BP, editor. Large Animal Internal Medicine, 2nd edition. New York: Mosby. 434-439. 
14. Radostitis OM, Gay CC, Blood DC, Hinchcliff KW (2000) Diseases caused by viruses and chlamydia II. In Radostitis OM, Gay CC, Blood DC, editors. Veterinary Medicine: A Textbook of the Diseases of Cattle Horses Sheep Pigs and Goats, 9th edition. Philadelphia: WB Saunders Co. 11351260.

15. Morris DD, Johnston JK (2002) Alterations in blood proteins. In Smith BP, editor. Large Animal Internal Medicine, 2nd edition. New York: Mosby. 427-433.

16. Carlson GP (2002) Clinical chemistry tests. In Smith BP, editor. Large Animal Internal Medicine, 2nd edition. New York: Mosby. 389-414.

\section{Corresponding author}

Dr. S.M. Abutarbush, B.V.Sc., M.Vet.Sc, Diplomate ABVP, Diplomate ACVIM

Department of Veterinary Clinical Sciences

Faculty of Veterinary Medicine

Jordan University of Science and Technology

Irbid 22110, Jordan

Phone: + 962796620635

Email: sameeh75@hotmail.com

Conflict of interests: No conflict of interests is declared. 